\title{
Front Matter: Volume 9426
}

, "Front Matter: Volume 9426," Proc. SPIE 9426, Optical Microlithography XXVIII, 942601 (24 April 2015); doi: 10.1117/12.2193695

SPIE. Event: SPIE Advanced Lithography, 2015, San Jose, California, United States 


\section{PROCEEDINGS OF SPIE}

\section{Optical Microlithography XXVIII}

Kafai Lai

Andreas Erdmann

Editors

24-26 February 2015

San Jose, California, United States

Sponsored by

Cymer, an ASML company (United States)

Published by

SPIE 
The papers included in this volume were part of the technical conference cited on the cover and title page. Papers were selected and subject to review by the editors and conference program committee. Some conference presentations may not be available for publication. The papers published in these proceedings reflect the work and thoughts of the authors and are published herein as submitted. The publisher is not responsible for the validity of the information or for any outcomes resulting from reliance thereon.

Please use the following format to cite material from this book:

Author(s), "Title of Paper," in Optical Microlithography XXVIII, edited by Kafai Lai, Andreas Erdmann, Proceedings of SPIE Vol. 9426 (SPIE, Bellingham, WA, 2015) Article CID Number.

ISSN: 0277-786X

ISBN: 9781628415285

Published by

SPIE

P.O. Box 10, Bellingham, Washington 98227-0010 USA

Telephone +1 3606763290 (Pacific Time) · Fax +1 3606471445

SPIE.org

Copyright $@ 2015$, Society of Photo-Optical Instrumentation Engineers.

Copying of material in this book for internal or personal use, or for the internal or personal use of specific clients, beyond the fair use provisions granted by the U.S. Copyright Law is authorized by SPIE subject to payment of copying fees. The Transactional Reporting Service base fee for this volume is $\$ 18.00$ per article (or portion thereof), which should be paid directly to the Copyright Clearance Center (CCC), 222 Rosewood Drive, Danvers, MA 01923. Payment may also be made electronically through $\mathrm{CCC}$ Online at copyright.com. Other copying for republication, resale, advertising or promotion, or any form of systematic or multiple reproduction of any material in this book is prohibited except with permission in writing from the publisher. The CCC fee code is $0277-786 \mathrm{X} / 15 / \$ 18.00$.

Printed in the United States of America.

Publication of record for individual papers is online in the SPIE Digital Library.

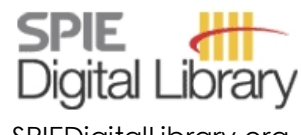

SPIEDigitalLibrary.org

Paper Numbering: Proceedings of SPIE follow an e-First publication model, with papers published first online and then in print. Papers are published as they are submitted and meet publication criteria. A unique citation identifier (CID) number is assigned to each article at the time of the first publication. Utilization of CIDs allows articles to be fully citable as soon as they are published online, and connects the same identifier to all online, print, and electronic versions of the publication. SPIE uses a six-digit CID article numbering system in which:

- The first four digits correspond to the SPIE volume number.

- The last two digits indicate publication order within the volume using a Base 36 numbering

system employing both numerals and letters. These two-number sets start with 00, 01, 02, 03, 04, $05,06,07,08,09,0 A, 0 B \ldots$. 0Z, followed by 10-1Z, 20-2Z, etc.

The CID Number appears on each page of the manuscript. The complete citation is used on the first page, and an abbreviated version on subsequent pages. 


\title{
Contents
}

\author{
ix Authors \\ xiii Conference Committee
}

\section{KEYNOTE SESSION}

942602 Optical lithography with and without NGL for single-digit nanometer nodes (Keynote Paper) [9426-1]

\section{PUSHING OPTICAL LIMIT}

942604 Evolving optical lithography without EUV (Invited Paper) [9426-3]

942605 Mask 3D induced phase and the mitigation by absorber optimization (Invited Paper) [9426-4]

942606 Patterning process exploration of metal 1 layer in $7 \mathrm{~nm}$ node with 3D patterning flow simulations [9426-5]

IMAGE AND PROCESS CONTROL

942607 Impact of bandwidth on contrast sensitive structures for low k1 lithography [9426-6]

942608 Solution for high-order distortion on extreme illumination condition using computational prediction method [9426-7]

942609 Optimum Arfi light source bandwidth for 10nm node logic imaging performance [9426-8]

9426 OB Single lithography exposure edge placement model [9426-10]

NON-IC APPLICATIONS

9426 OC Multicolor, visible-light nanolithography (Invited Paper) [9426-11]

9426 OD Progresses in 300mm DUV photolithography for the development of advanced silicon photonic devices (Invited Paper) [9426-12]

9426 OE Double-sided diffractive photo-mask for sub-500nm resolution proximity i-line mask-aligner lithography [9426-13]

9426 OF Optimization methods for 3D lithography process utilizing DMD-based maskless grayscale photolithography system [9426-14] 
$9426 \mathrm{OH} \quad$ Mask-induced best-focus-shifts in DUV and EUV lithography [9426-16]

9426 Ol Intensity and phase fields behind phase shifting masks studied with high resolution interference microscopy [9426-17]

\section{MULTIPLE PATTERNING AND SMO}

9426 J Study of cut mask lithography options for sub-20nm metal routing [9426-18]

9426 OK Inverse lithography using sparse mask representations [9426-19]

9426 OL RET selection on state-of-the-art NAND flash [9426-20]

9426 OM Pixel-based ant colony algorithm for source mask optimization [9426-21]

9426 ON Low-contrast photoresist development model for OPC applications at 10nm node [9426-22]

\section{MASK AND WAFER TOPOGRAPHY MODELING}

942600 Characterizing the dependence of thick-mask edge effects on illumination angle using AIMS images (CYMER Best Student Paper Award in Microlithography) [9426-23]

9426 OP Accurate, full chip 3D electromagnetic field model for non-Manhattan mask corners [9426-24]

9426 0Q A pattern- and optics-independent compact model of Mask3D under off-axis illumination with significant efficiency and accuracy improvements [9426-25]

9426 OR Printing circuits with $4 \mathrm{~nm}$ feature size: similarities and differences between EUV and optical lithographies [9426-26]

9426 OS Rigorous wafer topography simulation for investigating wafer alignment quality and robustness [9426-27]

OPC AND MODELING

9426 OT Investigating deprotection-induced shrinkage and retro-grade sidewalls in NTD resists [9426-29]

9426 OU Alternative to ILT method for high-quality full-chip SRAF insertion [9426-30]

9426 OV Uncertainty aware site selection method for OPC model calibration [9426-31]

9426 OW Experiments using automated sample plan selection for OPC modeling [9426-32] 
9426 0X Optical proximity correction with hierarchical Bayes model [9426-33]

9426 OY Application of SEM-based contours for OPC model weighting and sample plan reduction [9426-34]

DFM (DESIGN AND LITHO OPTIMIZATION): JOINT SESSION WITH CONFERENCES 9426 AND 9427

942610 Hot spots prediction after etching process based on defect rate [9426-36]

942611 Hybrid OPC flow with pattern search and replacement [9426-37]

OVERLAY OPTIMIZATION: JOINT SESSION WITH CONFERENCES 9424 AND 9426

942612 Overlay improvement methods with diffraction based overlay and integrated metrology [9426-38]

942613 Intra-field overlay correction for illumination based distortion [9426-39]

942614 Wafer to wafer overlay control algorithm implementation based on statistics [9426-40]

TOOLINGS

942616 Immersion and dry scanner extensions for sub-10nm production nodes [9426-46]

942617 Latest performance of ArF immersion scanner NSR-S630D for high-volume manufacturing for $7 \mathrm{~nm}$ node [9426-42]

942618 New ArF immersion light source introduces technologies for high-volume $14 \mathrm{~nm}$ manufacturing and beyond [9426-43]

942619 Total lithography system based on a new application software plafform enabling smart scanner management [9426-44]

9426 1A Green solution: 120W ArF immersion light source supporting the next-generation multiple-pattering lithography [9426-45]

POSTERS: IMAGE AND PROCESS CONTROL

9426 1C Comparing the experimental resist pattern width with aerial image intensity in high-NA projection lens [9426-48]

9426 ID Advanced process characterization using light source performance modulation and monitoring [9426-49]

$9426 \mathrm{IE}$ Analytical analysis for impact of polarization aberration of projection lens on lithographic imaging quality [9426-80] 
9426 if Reducing the substrate dependent scanner leveling effect in low-k1 contact printing [9426-50]

9426 IG A fast and flexible library-based thick-mask near-field calculation method [9426-51]

9426 1H Focus shift impacted by mask 3D and comparison between Att. PSM and OMOG [9426-52]

POSTERS: MULTIPLE PATTERNING AND SMO

9426 iJ 120W ArF laser with high-wavelength stability and efficiency for the next-generation multiple-patterning immersion lithography [9426-54]

9426 1K Forbidden pitches: causes, source optimization, and their role in design rules [9426-55]

$9426 \mathrm{lL}$ Source optimization using particle swarm optimization algorithm in photolithography [9426-79]

POSTERS: NON-IC APPLICATIONS

9426 IM Advanced Mask Aligner Lithography (AMALITH) [9426-56]

POSTERS: OPC MODEL

942610 An improved virtual aberration model to simulate mask 3D and resist effects [9426-58]

9426 iP Evaluation of compact models for negative-tone development layers at 20/14nm nodes [9426-59]

9426 IQ Photoresist 3D profile related etch process simulation and its application to full chip etch compact modeling [9426-60]

9426 IR Resist profile modeling with compact resist model [9426-61]

9426 is Impacts of post OPC shapes on pattern [9426-62]

9426 IT Calibrating etch model with SEM contours [9426-78]

\section{POSTERS: OPTICAL PROXIMITY CORRECTION}

9426 1U 7nm logic optical lithography with OPC-Lite [9426-63]

9426 IV OPC solution by implementing fast converging methodology [9426-64] 
9426 IW The comparison of various strategies of setting up an OPC repair flow with respect to process window constraints [9426-65]

9426 1X Model-based Hot Spot Fixing (HSF) by using target point control function [9426-66]

9426 IY Sub-Resolution Assist Feature (SRAF) printing prediction using logistic regression [9426-67]

9426 iz Accurate and fast computation of transmission cross coefficients [9426-68]

942621 The study of lithography conditions to use advanced resist performance properly [9426-70]

POSTERS: TOOLINGS

942623 Modeling and simulation of the beam steering unit [9426-72]

942624 DUV ArF light source automated gas optimization for enhanced repeatability and availability [9426-73]

942625 Performance of ETC controller in high-volume production [9426-74]

942626 Enabling CoO improvement thru green initiatives [9426-75]

942627 New robust and highly customizable light source management system [9426-76]

942628 Extending green technology innovations to enable greener fabs [9426-77] 
Proc. of SPIE Vol. $9426942601-8$

Downloaded From: https://www.spiedigitallibrary.org/conference-proceedings-of-spie on 26 Apr 2023 Terms of Use: https://www.spiedigitallibrary.org/terms-of-use 


\title{
Authors
}

Numbers in the index correspond to the last two digits of the six-digit citation identifier (CID) article numbering system used in Proceedings of SPIE. The first four digits reflect the volume number. Base 36 numbering is employed for the last two digits and indicates the order of articles within the volume. Numbers start with 00, 01, 02, 03, 04, 05, 06, 07, 08, 09, 0A, OB...0Z, followed by 10-1Z, 20-2Z, etc.

\author{
Abdo, Amr, OW \\ Ackmann, Paul, OT \\ Adam, Kostas, OP \\ Affentauschegg, Cedric, 08 \\ Agganwal, Tanuj, 18, 24 \\ Alagna, Paolo, 07, 09, 1D \\ Allouti, Nacima, OD \\ An, Yongbae, $1 \mathrm{X}$ \\ Aoyama, Hajime, 21 \\ Apostol, Ştefan, OK, 1K, IZ \\ Axelrad, Valery, $1 \mathrm{U}$ \\ Babcock, Carl P., 11 \\ Bachmann, Michael, 06 \\ Bailey, Todd, OY \\ Barnola, Sébastien, OD \\ Barouch, Eytan, OR \\ Baselmans, Jan, 08, 09 \\ Batarseh, Fadi, 11 \\ Baudot, Charles, OD \\ Bérard-Bergery, Sébastien, OD \\ Bibby, T., 18 \\ Bornebroek, Frank, 16 \\ Botter, Herman, 16 \\ Bourgin, Yannick, OE \\ Bramati, Arianna, 1M \\ Brision, Stéphane, OD \\ Brunner, Timothy, 13 \\ Cacouris, T., 18 \\ Casati, Nathalie, OW \\ Chang, C. S., $1 F$ \\ Chen, Ao, $1 \mathrm{P}$ \\ Chen, K. C., IF \\ Chen, Quan, 21 \\ Chen, Xuanbo, $1 G$ \\ Choi, Jaeseung, $1 X$ \\ Choi, Young-Sin, 08, 12 \\ Chu, W. H., is \\ Chunder, Anindarupa, 0J \\ Chung, Angeline, IP \\ Ciofi, Ivan, 06 \\ Clifford, Chris, OP \\ Comboroure, Corinne, OD \\ Conley, Will, 07, 18, 25 \\ Connolly, Brid, 00 \\ Coskun, Tamer H., OV \\ Croffie, Ebo, $0 Q$ \\ Dao, Hoang, 25 \\ de Boeij, Wim P., 16 \\ de Graaf, Roelof F., 16
}

de Kort, Toine, 16

Demmerle, Wolfgang, 06

Deng, Yunfei, IP, IR

Do, Munhoe, $1 \mathrm{X}$

Dong, Lisong, $1 \mathrm{G}$

Dong, LiSong, $1 \mathrm{H}$

Droste, Richard, 16

Du, Yaojun, IV, IW

Dunlap, David, 25

Enami, Tatsuo, 27, 28

Erdmann, Andreas, $\mathrm{OH}$

Evanschitzky, Peter, $\mathrm{OH}$

Feng, Jiayun, 23

Ferrotti, Thomas, OD

Finders, Jo, 05

Flagello, Donis G., 04

Flores, Ronnie, 25

Foong, Yee Mei, 1P, 1Y

Fourkas, John T., OC

Fryer, David, OP, IP

Fujii, Koichi, 10

Fukuda, Hitomi, 28

Funatsu, Takayuki, 17

Gabrani, Maria, OW

Galvier, Jean, 05

Gao, Jie, 1G

Gao, Weimin, 06

Gavrilin, Dmitrii, OS

Gays, Fabien, OD

Geh, Bernd, 08

Ghaida, Rani S., 11

Gillespie, Walter, 26

Gillijns, Werner, 06

Granik, Yuri, 1P, 1R

Gräupner, Paul, $\mathrm{OH}$

Graur, loana, OY

Greiner, Andreas, OS

Gross, Eric, 18, 26

Guo, Moran, $1 \mathrm{H}$

Ha, Hun Hwan, 08, 12

Halle, Scott, OY

Haran, Don, 26

Hayakawa, Akira, 17

$\mathrm{He}, \mathrm{Xin}, 23$

$\mathrm{He}$, Yuan, $\mathrm{OL}$

Herzig, Hans Peter, 0

Hikida, Yujiro, 17

Hirai, Yoshikazu, OF

Hirayama, Toru, 17 
Hitomi, Keiichiro, OY

Hofmann, Detlef, OS

Hou, Yaching, 07

Hsieh, Simon, 07

Huang, C. H., IF

Huang, S. Y., is

Hurley, Paul, OK, 1K, 12

Hwang, Hyun Woo, 14

Imai, Motokatsu, 10

Ionescu, Radu-Cristian, OK, 1 Z

Ishida, Keisuke, 1 A, $1 \mathrm{~J}$

Ishii, Hiroyuki, IU

Ishiyama, Satoshi, 17

Jaiswal, Om, OW

Janda, Eric, 08

Jantzen, Kenneth, $1 T$

Jing, Nan, 13

Kakizaki, Kouji, 1A, $1 \mathrm{~J}$

Kamohara, Itary, OS

Kanaya, Reiji, 10

Kang, Young-Seog, 08, 12, 14

Käsebier, Thomas, OE

Kato, Yoshiki, OF

Kim, Sunny Jang-Sun, 08, 12

Kim, Ryoung-Han, 0J

Kim, Seongjin, $1 \mathrm{X}$

Kim, Young Ha, 08

Kim, Young Hoon, 12

Kimura, Taiki, 10

Kley, Ernst-Bernhard, OE

Klostermann, Ulrich, OS

Koh, Kar Kit, IY

Kong, Jeong Heung, 12, 14

Kono, Hirotaka, 17, 19

Kopp, Christophe, OD

Kotani, Toshiya, 10

Kumazaki, Takahito, 1A, $1 \mathrm{~J}$

Kuo, C. C., IS

Kuo, Hung-Fei, OM

Kurosu, Akihiko, 1A, 1J

Kwon, Oh-Sung, 08

Kye, Jongwook, OJ, OT

Lafferty, Neal, OL

Lake, Matt, 25, 26

Lam, Michael, OP

Lange, Falk, 00

Lee, Byeong Soo, 14

$\mathrm{Li}$, Frederick, OM

Li, Sikun, 1E, $1 \mathrm{~L}$

Li, Xiaoping, 23

Li, Yanqiu, $1 \mathrm{G}$

Lin, Burn J., 02

Lin, H. T., 1S

Lin, Qun Ying 10

Liv, QingWei, OL

Liu, Yansong, $1 \mathrm{H}$

Luan, Lan, 1Q

Lucas, Kevin, 06

Lutich, Andrey, OU

Ma, Xiaoxu, OF
$M a, X u, 1 G$

Madhavan, Sriram, 11

Mailfert, Julien, 09

Mariolle, Denis, OD

Martinez, Pedro, 07

Masaki, Kazuo, 19

Mason, Eric, 26

Matagne, Philippe, 06

Matsunaga, Takashi, 1A, $1 \mathrm{~J}$

Matsunawa, Tetsuaki, OX

Matsuyama, Tomoyuki, 19, 10, 21

McGowan, Sarah, 11

McLaren, Matthew G., 16

Medvedev, Dmitry, IP

Mehta, Sohan, OT

Melchior, J., 18

Menezo, Sylvie, OD

Mikami, Koji, $1 U$

Miller, Marshal, OY

Minegishi, Yuji, 27, 28

Miyazawa, Tami, 21

Mizoguchi, Hakaru, 1A, $1 \mathrm{~J}$

Moeller, Holger, OS

Morgana, Nicoló, OS

Morgenfeld, Bradley, 13

Nakashima, Toshiharu, 1C

Nakayama, Ryo, $1 \mathrm{U}$

Nam, Young-Sun, 08, 12

Neumann, Jens Timo, $\mathrm{OH}$

Neureuther, Andy, 00

Oberschmidt, James, OW

O'Brien, Kevin, 24, 25

Ochiai, Hideyuki, 27

Ohta, Takeshi, 1A, 1 J, 27

Oliver, Mike, OP

Omran, A., $1 T$

Oulmane, Mohamed, 06

Oyama, Kenichi, $1 \mathrm{U}$

Padmabandu, G. G., 26

Pan, David Z., OX

Park, Seemoon, 19

Pathak, Piyush, 11

Pei, Jinhua, OL

Pike, Michael, 13

Ping, Yang Y., 11

Pistor, Thomas V., OT

Ponomarenko, Mariya, 08

Preuninger, Juergen, OS

Puthankovilakam, Krishnaparvathy, 0l

Rechtsteiner, Greg, 09, 1D

Renwick, Stephen P., 04

Rizvi, Umar, 08

Saad, Yves, 06

Scharf, Toralf, 0 l

Schmoeller, Thomas, 06

Sczyrba, Martin, 00

Sei, Toshihiko, 21

Shanker, Aamod, 00

Shao, Chun, 21

Shao, Feng, OL 
Shen, Lina, 1E

Shi, Xuelong, OL

Shibazaki, Yuichi, 17, 19

Shibuya, Masato, 1C

Shin, Ju Hee, 08, 12

Shin, Si Woo, 12

Shirata, Yosuke, 17

Siefke, Thomas, OE

Simic, Aleks, 25

Slotboom, Daan, 08

Smayling, Michael C., $1 \mathrm{U}$

Somani, Shikha, 11

Song, Hua, ON, IQ

Song, Myeong Gyu, 14

Song, Zhiyang, $1 \mathrm{H}$

Souhaité, Aurélie, OD

Su, Xiaojing, $1 \mathrm{H}$

Su, Yajuan, $1 \mathrm{H}$

Sugihara, Taro, 19

Szelag, Bertrand, OD

Tabata, Osamu, OF

Takada, Akira, 1C

Takahisa, Kenji, 27, 28

Takemasa, Kengo, 21

Tan, Chin Boon, IY

Tejnil, Edita, OP

Thornes, Joshua, 18, 25

Timoshkov, Vadim, 09

Tomova, Zuleykhan, OC

Tsai, Y. T., $1 \mathrm{~S}$

Tseng, C. F., IF

Tsuchiya, Toshiyuki, OF

Tsujita, Koichiro, $1 \mathrm{U}$

Tsushima, Hiroaki, 1A, 1J

Tsuzuki, Takao, 10

Tyminski, Jacek K., OB

Uehara, Yusaku, 17

Ujazdowski, Richard, 26

van Ballegoij, Rob, 16

van der Heijden, Rob W., 08

van der Sanden, Stefan, 08

van Kempen, Floris, OF

van Keulen, Fred, OF

Verma, Piyush, 11

Viswanathan, Ramya, OW

Vizioz, Christian, OD

Voelkel, Reinhard, OI, 1M

Vogler, Uwe, OI, IM

Wakamoto, Shinji, 19

Waller, Laura, 00

Wang, Chenchen, OJ, OT

Wang, Jinchun, 23

Wang, Jingyu, 11

Wang, Lei, 1L

Wang, Wuping, 21

Wang, Xiangzhao, 1E, 1L

Wang, Yan, OJ, OT

Watanabe, Yuki, 10

Watts, Josef, OW

Wehrung, Brian, 25
Wei, David, ON, OQ

Wei, Yayi, $1 \mathrm{H}$

Weichelt, Tina, ol

Weichselbaum, Stefan, 16

Weisbuch, François, IT

Wiltshire, Timothy, 13

Wong, Patrick, 09

Woo, Youngtag, OJ

Wu, Cheng-En, ON, IQ

Wu, Wei-Chen, OM

Wu, Yixu, OT

Wyman, John, 25

Yaegashi, Hidetami, $1 \mathrm{U}$

Yamauchi, Shohei, $1 \mathrm{U}$

Yan, Guanyong, 1E, 1L

Yan, Qiliang, $0 Q$

Yang, Chaoxing, $1 \mathrm{~L}$

Yang, Elvis, IF

Yang, Hyunjo, $1 \mathrm{X}$

Yang, Qing, IV, IW

Yang, T. H., IF

Yang, Wayne, 1Q

Yang, Zhengkai, 21

Yeung, Michael S., OR

Yim, Donggyu, $1 \mathrm{X}$

Yoo, Youngsun, 28

Yu, Bei, OX

Yuan, Lei, OJ, OT

Yun, Sang Ho, 12

Zeitner, Uwe D., OE, Ol

Zeng, Jia, OJ

Zhang, Charlie, ON

Zhang, Dong Qing, IP, IY

Zhang, Hongbo, $O Q$

Zhang, Hongxin, IP

Zuniga, Christian, IR

Zurita, Omar, 09, 1D 
Proc. of SPIE Vol. $9426942601-12$

Downloaded From: https://www.spiedigitallibrary.org/conference-proceedings-of-spie on 26 Apr 2023 Terms of Use: https://www.spiedigitallibrary.org/terms-of-use 


\section{Conference Committee}

Symposium Chair

Mircea V. Dusa, ASML US, Inc. (United States)

Symposium Co-chair

Bruce W. Smith, Rochester Institute of Technology (United States)

Conference Chair

Kafai Lai, IBM Corporation (United States)

Conference Co-chair

Andreas Erdmann, Fraunhofer-Institut für Integrierte Systeme und Bavelementetechnologie IISB (Germany)

Conference Program Committee

Pary Baluswamy, Micron Technology, Inc. (United States)

Peter D. Brooker, Synopsys, Inc. (United States)

Will Conley, Cymer, Inc. (United States)

Jo Finders, ASML Netherlands B.V. (Netherlands)

Carlos Fonseca, Tokyo Electron America, Inc. (United States)

Tsai-Sheng Gau, Taiwan Semiconductor Manufacturing Company Ltd. (Taiwan)

Bernd Geh, Carl Zeiss SMT Inc. (United States)

Yuri Granik, Mentor Graphics Corporation (United States)

Young Seog Kang, SAMSUNG Electronics Company, Ltd.

(Korea, Republic of)

Sachiko Kobayashi, Toshiba Corporation (Japan)

Jongwook Kye, GLOBALFOUNDRIES Inc. (United States)

Soichi Owa, Nikon Corporation (Japan)

John S. Petersen, Periodic Structures, Inc. (United States)

Daniel Sarlette, Infineon Technologies Dresden (Germany)

Xuelong Shi, Semiconductor Manufacturing International

Corporation (China)

Sam Sivakumar, Intel Corporation (United States)

Bruce W. Smith, Rochester Institute of Technology (United States)

Kazuhiro Takahashi, Canon Inc. (Japan)

Geert Vandenberghe, IMEC (Belgium)

Reinhard Voelkel, SUSS MicroOptics SA (Switzerland) 


\section{Session Chairs}

1 Keynote Session

Kafai Lai, IBM Corporation (United States)

Andreas Erdmann, Fraunhofer-Institut für Integrierte Systeme und Bavelementetechnologie IISB (Germany)

2 Pushing Optical Limit

Bernd Geh, Carl Zeiss SMT Inc. (United States)

Will Conley, Cymer LLC (United States)

3 Image and Process Control

Kazuhiro Takahashi, Canon Inc. (Japan)

Carlos Fonseca, Tokyo Electron America, Inc. (United States)

4 Non-IC Applications

Andreas Erdmann, Fraunhofer-Institut für Integrierte Systeme und

Bavelementetechnologie IISB (Germany)

Reinhard Völkel, SUSS MicroOptics SA (Switzerland)

5 Mask Topography: Joint Session with Conferences 9422 and 9426

Ted Liang, Intel Corporation (United States)

Hsu-Ting Huang, Taiwan Semiconductor Manufacturing Company

Ltd. (Taiwan))

6 Multiple Patterning and SMO

Xuelong Shi, Semiconductor Manufacturing International Corporation (China)

Emily Gallagher, IMEC (Belgium)

7 Mask and Wafer Topography Modeling

John S. Petersen, Periodic Structures, Inc. (United States)

Young Seog Kang, SAMSUNG Electronics Company, Ltd.

(Korea, Republic of)

8 OPC and Modeling

Yuri Granik, Mentor Graphics Corporation (United States)

Peter D. Brooker, Synopsys, Inc. (United States)

9 DFM (Design and Litho Optimization): Joint Session with Conferences 9426 and 9427

Jongwook Kye, GLOBALFOUNDRIES Inc. (United States)

Andrew R. Neureuther, University of California, Berkeley (United States)

10 Overlay Optimization: Joint Session with Conferences 9424 and 9426

John C. Robinson, KLA-Tencor Corporation (United States)

Mark Phillips, Intel Corportation (United States) 
11 DSA Design for Manufacturability: Joint Session with Conferences 9423, 9426, and 9427

Michael A. Guillorn, IBM Thomas J. Watson Research Center (United States)

Sachiko Kobayashi, Toshiba Corporation (Japan)

Vivek K. Singh, Intel Corporation (United States)

12 Toolings

Soichi Owa, Nikon Corporation (Japan)

Daniel Sarlette, Infineon Technologies Dresden (Germany) 
Proc. of SPIE Vol. $9426942601-16$

Downloaded From: https://www.spiedigitallibrary.org/conference-proceedings-of-spie on 26 Apr 2023 Terms of Use: https://www.spiedigitallibrary.org/terms-of-use 